\title{
A single cell transcriptional roadmap for cardiopharyngeal fate diversification
}

\author{
Wei Wang ${ }^{1, \#}$, Xiang Niü2,3,5,\#, Tim Stuart ${ }^{3}$, Estelle Jullian" ${ }^{4}$, William Mauck ${ }^{3}$, Robert G. Kelly , \\ Rahul Satija ${ }^{2,3,{ }^{*}}$ and Lionel Christiaen ${ }^{1, *}$
}

\begin{abstract}
In vertebrates, multipotent progenitors located in the pharyngeal mesoderm form cardiomyocytes and branchiomeric head muscles, but the dynamic gene expression programs and mechanisms underlying cardiopharyngeal multipotency and heart vs. head muscle fate choices remain elusive. Here, we used single cell genomics in the simple chordate model Ciona, to reconstruct developmental trajectories forming first and second heart lineages, and pharyngeal muscle precursors, and characterize the molecular underpinnings of cardiopharyngeal fate choices. We show that FGF-MAPK signaling maintains multipotency and promotes the pharyngeal muscle fate, whereas signal termination permits the deployment of a pan-cardiac program, shared by the first and second lineages, to define heart identity. In the second heart lineage, a Tbx1/10-Dach pathway actively suppresses the first heart lineage program, conditioning later cell diversity in the beating heart. Finally, cross-species comparisons between Ciona and the mouse evoke the deep evolutionary origins of cardiopharyngeal networks in chordates.
\end{abstract}

Distinct cell types form multicellular animals and execute specialized functions within defined organs and systems, implying that individual cells within progenitor fields must acquire both organ-level and cell-type-specific identities. The mammalian heart comprises chamber-specific cardiomyocytes, various endocardial cell types, fibroblasts and smooth muscles $^{1}$, and despite their specialized features, these cells share a cardiac identity. Popular models posit that heart cells emerge from multipotent cardiovascular progenitors ${ }^{2}$, implying that multipotent progenitors are first imbued with a cardiac identity, before producing a diversity of cell types. Consistent with this model, mammalian heart cells emerge primarily from Mesp1+ mesodermal progenitors ${ }^{3-5}$. However, lineage tracing and clonal analyses indicated that distinct compartments arise from separate progenitor pools, referred to as the first and second heart fields ${ }^{6-11}$. In addition, most early cardiac progenitors produce only one cell type ${ }^{12}$, and cell type segregation occurs early, possibly prior to commitment to a heart identity ${ }^{13}$. Moreover, derivatives of the second heart field (e.g. cardiomyocytes of the right ventricle and outflow tract) share a common origin with branchiomeric head muscles, in the cardiopharyngeal

\footnotetext{
${ }^{1}$ Center for Developmental Genetics, Department of Biology, New York University, New York, NY, USA

${ }^{2}$ Center for Genomics and Systems Biology, Department of Biology, New York University, New York, NY, USA

${ }^{3}$ New York Genome Center, New York, NY, USA

${ }^{4}$ Aix-Marseille University, CNRS UMR 7288, Developmental Biology Institute of Marseille, Campus De Luminy Case 907,13288 Marseille Cedex 9, France

${ }^{5}$ present address: Tri-Institutional Program in Computational Biology and Medicine, Weill Cornell Medical College, New York, New York, USA

\#: contributed equally to the work

*: corresponding authors: Ic121@nyu.edu (L.C.), rsatija@nygenome.org (R.S.)
} 
mesoderm ${ }^{4,14-21}$. The characteristics of multipotent cardiopharyngeal progenitors, and the mechanisms underlying early heart vs. pharyngeal/branchiomeric muscle fate choices remain largely elusive, and studies are partially hindered by the complexity of vertebrate embryos ${ }^{21,22}$.

The tunicate Ciona emerged as an innovative chordate model to study cardiopharyngeal development with unprecedented spatio-temporal resolution. In Ciona, invariant cell divisions produce distinct first and second heart lineages, and pharyngeal muscle precursors from defined multipotent cardiopharyngeal progenitors ${ }^{23,24}$ (Fig. 1a). Multipotent progenitors exhibit multilineage transcriptional priming, whereby conserved fate-specific determinants are transiently co-expressed, before regulatory cross-antagonisms partition the heart and pharyngeal muscle programs to their corresponding fate-restricted precursors ${ }^{23-25}$. Here, we characterised, with single cell resolution, the genome-wide characteristics and regulatory mechanisms governing cardiopharyngeal multipotency, early fate choices, and the establishment of cell diversity in the beating heart.

\section{RESULTS}

\section{Single cell transcriptome profiling of early cardiopharyngeal lineages}

To characterize gene expression changes underlying the transitions from multipotent progenitors to distinct fate-restricted precursors, we performed plate-based single cell RNA sequencing (scRNA-seq) with SMART-Seq $2^{26}$ on cardiopharyngeal-lineage cells FACS-purified from synchronously developing embryos and larvae (Fig. 1a). We obtained 848 high-quality single cell transcriptomes from 5 time points covering early cardiopharyngeal development (Fig. 1a, Extended Data Fig. 1d). Using an unsupervised strategy ${ }^{27}$, we clustered single cell transcriptomes from each time point, and identified clusters according to known markers and previously established lineage information (Fig. 1a-b, Extended Data Fig. 1a, c). Focusing on fate-restricted cells isolated from post-hatching larvae (18 and 20 hours post-fertilization (hpf), FABA stages 26-28; Extended Data Table 1), we identified clusters of Gata4/5/6+ first and second heart precursors, and Ebf+ atrial siphon muscle (ASM) precursors ${ }^{24,25,28}$ (Fig. 1b). Differential expression analyses identified (1) ASM/pharyngeal muscle vs. pan-cardiac specific markers and (2) first vs. second heart precursor-specific markers (Extended Data Fig. 1b, Extended Data Table 2). The top 111 predicted pan-cardiac genes comprised established cardiac determinants, including Gata4/5/6, Nk4/Nkx2-5, and Hand, and we confirmed heart-specific expression by fluorescent in situ hybridization (FISH) for 19 candidate markers, including Meis and Lrp4/8 (Extended Data Fig. 1e,g; Extended Data Table 2, 3). The pan-cardiac vs. pharyngeal muscle contrast dominated late cellular heterogeneity, but first and second heart precursor populations also segregated (Fig. 1b, Extended Data Fig. 1a), revealing 18 and 7 first- and second-lineage-specific markers, respectively (e.g. Mmp21 and Dach; Extended Data Fig. 1f; Extended Data Tables 2,3). Our analyses thus uncovered specific programs activated in fate-committed progenitors, including both shared ('pan-cardiac') and first- vs. second-lineage-specific signatures for heart precursors.

To characterize gene expression dynamics, we ordered single cell transcriptomes from successive time points on pseudotemporal developmental projections ${ }^{29,30}$. Using the whole dataset while ignoring established clonality, we identified multipotent progenitors and separate 
cardiac and pharyngeal muscles branches (Extended Data Fig. 2). However, this unsupervised analysis failed to correctly distinguish the first and second heart lineages, likely because the shared pan-cardiac program dominates lineage-specific signatures (Extended Data Fig. 1b). Taking advantage of the invariant lineage (Fig. 1a), we combined cells corresponding to each branch, and created three unidirectional trajectories representing first- and second heart, and pharyngeal muscle lineages (Fig. 1c, Extended Data Fig. 3a-b). The distribution of cells along pseudotime axes corresponded to the time points of origin (Fig. 1c, Extended Data Fig. 3b, g; average Pearson Correlation Coefficient, PCC $=0.889$ ), while providing higher-resolution insights into the gene expression dynamics. Validating this approach, in silico trajectories captured known lineage-specific expression changes of cardiopharyngeal regulators (Fig. 1d, Extended Data Fig. 3c-d; ${ }^{24,25,31}$ ).

Developmental trajectories suggest a continuous process marked by gradual changes in gene expression. However, the latter occur preferentially in defined 'pseudotime' windows for multiple genes (Extended Data Fig. 3e-f), consistent with more abrupt biological transitions, such as cell divisions ${ }^{31}$. To identify possible switch-like discontinuities, we determined cell-to-cell cross-correlations along lineage-specific trajectories. Using constrained hierarchical clustering $^{32}$, we identified 10 putative discrete regulatory states across the cardiopharyngeal trajectories, including two multipotent states, and eight successive transitions towards fate restriction (Fig. 1e, Extended Data Fig. 3h).

These successive transitions reveal underlying lineage-specific transcriptional dynamics. For example, the multipotent cardiopharyngeal progenitor state (aka TVC) differed markedly from subsequent cardiac states along the first cardiac trajectory (Fig. 1e), and gene expression mapping distinguished 'primed' and 'de novo'-expressed heart markers, such as Gata4/5/6 and Slit1/2/3, respectively (Fig. 1d). Conversely, primed pharyngeal muscle markers were downregulated along cardiac trajectories, as expected (Extended Data Fig. 3c, e-f; ${ }^{33,34}$ ). Multilineage transcriptional priming is a hallmark of cardiopharyngeal multipotency that remained to be characterized globally ${ }^{25}$. Here, we estimated that $41 \%(73 / 176)$ of the pharyngeal-muscle-specific and 53\% (59/111) of the pan-cardiac markers are already expressed in multipotent progenitors, indicating that lineage-specific maintenance of primed genes is a major determinant of cell-type-specific transcriptomes in the cardiopharyngeal lineage. Nevertheless, $88 \%(3,504 / 3,982)$ of late-expressedtranscripts, were already detected in multipotent progenitors (Fig. 1f), showing that de novo cell-type-specific gene activation contributes significantly to cell-type-specific programs (Fisher's exact test, $P<2.2 \times 10^{-16}$ for both the pan-cardiac- and ASM-specific gene sets, respectively).

We further explored the molecular basis for progression through regulatory states. As a proof of concept, we first focused on the pharyngeal muscle trajectory, for which the key regulators Hand-r, Tbx1/10 and Ebf have been characterized ${ }^{24,25,28,31,35}$ (Extended Data Figure 4). The first two regulatory states corresponded to successive generations of multipotent cardiopharyngeal progenitors (aka TVCs and STVCs, Fig. 1a, Extended Data Fig. 3g-i), confirming that asymmetric cell divisions provide the biological basis for these first transitions. To our surprise, the majority of newborn pharyngeal muscle precursors isolated from $16 \mathrm{hpf}$ larvae clustered with multipotent progenitors isolated from 14hpf embryos (aka STVCs, Fig. 1a, 
Extended Data Fig. 3g-i), although they already expressed Ebf (Extended Data Fig. 4a), as previously observed by FISH $^{24,31}$. This indicates that, although newborn pharyngeal muscle progenitors already express a key determinant, their transcriptome remains similar to their multipotent mother cells. Indeed, the pharyngeal muscle transcriptome is progressively remodelled as cells transition through successive states, involving both downregulation of primed cardiac markers and 'de novo' activation of pharyngeal muscle markers (Extended Data Fig. 4a-c, h-j). Moreover, systematic comparison with expression profiles following perturbations of Ebf function ${ }^{25}$ indicated that candidate Ebf target genes, including Myogenic regulatory factor (Mrf) (the MyoD/Myf5 homolog), and Myosin heavy chain 3 (Mhc3), are activated at later timepoints, consistent with Ebf-dependent transitions to committed pharyngeal muscle states ${ }^{31}$ (Extended Data Fig. 4a, d, e-g, k).

\section{Termination of FGF-MAPK signaling launches a pan-cardiac program for heart identity}

Next we investigated gene expression changes underlying state transitions during cardiac specification. We focused on the first heart trajectory, which provided the largest pseudo-temporal range, to characterize the pan-cardiac program. Activation of 'de novo' pan-cardiac markers and down-regulation of primed pharyngeal muscle and multipotent-specific markers accounted for most gene expression changes (Fig. 2a-b, Extended Data Fig. 5a-d). These coordinated gene expression changes explained major transitions along the cardiac trajectories. For example, we used logistic regression to determine that Slit1/2/3 is activated at the transition between the multipotent and FHP1 states, which we confirmed by FISH (Fig. 1d, Extended Data Fig. 1g). We identified a principal component (PC1), which correlated highly with pseudotime $(\mathrm{PCC}=0.96$; Extended Data Fig. 5e), and used the PC1-loading of each gene to estimate the relative contribution of each class of markers to discrete regulatory states (Fig. 2c). This suggested that the multipotent state is primarily determined by the combined expression of TVC-specific genes, and primed cardiac and pharyngeal muscle markers. The TVC-to-FHP1 transition is marked by a sharp decline in TVC-specific gene expression, accompanied by down-regulation of primed ASM genes, upregulation of primed pan-cardiac genes, and activation of de novo-expressed pan-cardiac genes. In this regard, the FHP1 state may be considered a "transition state" between a multipotent TVC state, and the FHP2 state $^{36}$. The latter is defined by the virtual absence of TVC-specific and primed ASM-specific transcripts, and high levels of both primed and de novo-expressed pan-cardiac markers, thus probably corresponding to a heart-specific state, whereas activation of cell-type/lineage-specific genes underlie the FHP2-to-FHP3 transition, and their expression helps define the first-lineage-specific state, $\mathrm{FHP}_{3}$, as is the case for Mmp21 (Fig. 2d, Extended Data Fig. 5f).

A true pan-cardiac program should unfold following similar dynamics in the first and second heart lineage, reflecting shared regulatory logic. Accordingly, we observed a striking agreement between the ordered activation pattern of individual genes along each trajectory (Fig. 2e), suggesting a remarkably conserved developmental program. Notably, the onset of each gene was consistently delayed in the second heart trajectory, starting with the STVC-to-SHP1 transition, as second heart precursors are born from a second generation of multipotent progenitors, $\sim 2 \mathrm{~h}$ later than first heart precursors (Fig. 1a, 2e, f). Therefore, the de novo pan-cardiac program is 
tightly regulated and deployed in a reproducible cascade, whose onset is independently induced in the first and second heart lineages as they arise from multipotent progenitors.

We then sought to identify regulatory switches triggering the full pan-cardiac program in heart lineages. The FGF-MAPK signaling pathway is active and maintained specifically in multipotent cardiopharyngeal progenitors (TVCs and STVCs), and in early pharyngeal muscle precursors (ASMF), where it promotes the expression of Hand-r, Tbx1/1O, and Ebf. By contrast, signaling is terminated in newborn first and second heart precursors ${ }^{31}$. We integrated sc- and bulk RNA-seq performed on FACS-purified cardiopharyngeal lineage cells following defined perturbations, and determined that FGF-MAPK signaling opposed pan-cardiac gene expression, while promoting the pharyngeal muscle program in swimming larvae (18 and $20 \mathrm{hpf}$, Fig. 2g-h, Extended Data Fig. 5j-m, Extended Data Table 4). At earlier stages (12 and 15 hpf), FGF-MAPK perturbations generally did not affect the expression of primed pan-cardiac genes in multipotent progenitors, whereas de novo-expressed genes were upregulated upon signaling inhibition by Fgfr $^{\text {DN }}$ misexpression (Fig. 2g-h, Extended Data Fig. 5h-i,n-q; Extended Data Table 4). FISH assays further demonstrated that the de novo-expressed pan-cardiac marker Lrp4/8 was upregulated in TVCs upon misexpression of $\mathrm{Fgfr}^{\mathrm{DN}}$ (Fig. 2i). These analyses indicated that heart-lineage-specific termination of FGF-MAPK signaling permits the activation of de novo-expressed pan-cardiac genes, and subsequent heart fate specification, whereas ongoing FGF-MAPK signaling in cardiopharyngeal progenitors promotes multipotency both by maintaining the primed pharyngeal muscle program and by inhibiting the full deployment of the heart-specific program (Extended Data Fig. 12).

\section{Differences between cardiac lineages foster cellular diversity in the beating heart}

A shared pan-cardiac gene program progressively defines the heart identity, but distinct precursors nevertheless clustered separately, revealing significant differences between the first and second heart lineages (Fig. 1b, Extended Data Fig. 1b; Extended Data Table 2). We mined the second heart trajectory to explore the development and evolution of the vertebrate second heart field (SHF), since the ascidian and vertebrate SHFs share regulatory inputs from Nk4/Nkx2-5 and Tbx1/1O orthologs s $^{11,14,24,37-41}$. Examining our list of markers distinguishing second and first heart precursors, we identified the dachshund homolog Dach as the only known transcription regulator ${ }^{42-45}$ (Extended Data Fig. 1f, 6a; Extended Data Table 2), and its upregulation as cells transitioned from a multipotent state suggested a role in specifying the second cardiac identity (Fig. 3a).

Dach1 and -2 have not been previously implicated in mammalian SHF development, but they belong to the conserved "retinal network" 46 , which comprises homologs of Six and Eya transcription factors that contribute to cardiopharyngeal development in the mouse ${ }^{47,48}$. Lineage-specific CRISPR/Cas9-mediated loss-of-function ${ }^{49,50}$, followed by gene expression assays, indicated that Dach is neither required for activation of the pharyngeal muscle marker $E b f$, nor for its exclusion from the SHPs (Extended Data Fig. 6b). By contrast, loss of Dach function caused ectopic expression of the FHP marker Mmp21 in the second heart precursors (Fig. 3b,c, Extended Data Fig. 6c). A CRISPR-resistant Dach ${ }^{\text {PAMmut }}$ cDNA rescued the ectopic Mmp21 expression in the second heart lineage, but did not abolish endogenous Mmp21 expression in the first heart lineage (Fig. 3c, Extended Data Fig. 6c). Dach is thus both a marker 
and a key regulator of second heart lineage specification, which is required, but not sufficient, to prevent activation of the first-lineage-specific marker Mmp21.

Since the second heart lineage emerges from $T b x 1 / 1 O+$ multipotent progenitors ${ }^{24}$, we tested whether Tbx1/10 regulates Dach expression. CRISPR/Cas9-mediated lineage-specific loss of Tbx1/10 function ${ }^{35}$ inhibited Dach expression (Fig. 3d, Extended Data Fig. 6d), and caused ectopic activation of Mmp21 (Fig. 3c, Extended Data Fig. 6b), indicating that Tbx1/10 promotes second heart lineage specification, in part by regulating Dach activation, in addition to its role in pharyngeal myogenesis.

Indeed, Tbx1/10 is also necessary in parallel to FGF-MAPK activity to activate $E b f$ and promote the pharyngeal muscle program ${ }^{24,31,35}$, in a manner similar to Tbx1 function in vertebrate branchiomeric myogenesis ${ }^{51,52}$. To explore the mechanism distinguishing between Tbx1/10 dual functions, we used the MEK/MAPKK inhibitor Uo126, which inhibits Ebf expression $^{31}$, and caused ectopic Dach activation in the lateral-most cardiopharyngeal cells that normally form $E b f+$ pharyngeal muscle precursors (Fig. 3e). Moreover, Tbx1/10 misexpression and MEK/MAPKK inhibition synergized to cause precocious and ectopic Dach activation in cardiopharyngeal progenitors (Extended Data Fig. 6e). Taken together, these data indicate that termination of FGF-MAPK signaling in $T b x 1 / 1 O+$ cardiopharyngeal progenitors suffice to activate Dach expression and promote the second heart lineage identity, and demonstrate how distinct signaling environments can promote divergent regulatory programs in concert with Tbx1/10 expression.

First and second heart precursors share a common pan-cardiac signature, but initial molecular differences open the possibility that each lineage contributes differently to cardiogenesis. The beating Ciona heart is demonstrably simpler than its vertebrate counterpart; yet, diverse cell types form its single U-shaped compartment ${ }^{53}$. In post-metamorphic juveniles, the heart already beats, and double labeling with a Mesp>nls::lacZ reporter and the cardiac-specific myosin heavy chain 2 (Mhc2/Myh6) marker showed that beta-galactosidase+; Mhc2/Myh6- cells surround Mhc2/Myh6+ cardiomyocytes ${ }^{24,28}$. Lineage tracing using the photoconvertible reporter Kaede ${ }^{25}$ indicated that first and second heart precursors derivatives remain within largely separate domains in juvenile hearts (Fig. 3f, Extended Data Fig. 7a). Specifically, first-lineage-derived cells form the inner layer of Mhc2+ cardiomyocytes, whereas second-lineage-derived cells contribute to the outer layer of Mhc2- cells (Fig. 3g, Extended Data Fig. 7c, Extended Data Movie S1). Triple labeling and cell quantification using pan-cardiopharyngeal and second-heart-lineage-specific reporters, and the Mhc2 probe, indicated that most Mhc2+ cardiomyocytes were located in the first-lineage-derived inner layer, whereas only $\sim 17 \%$ of second-lineage-derived cells express Mhc2 in control juveniles (Fig. 3g). Thus, the first and second heart lineages contribute primarily Mhc2/Myh6+ cardiomyocytes and Mhc2/Myh6- cells to the beating juvenile heart, respectively.

To further characterize cellular diversity in the juvenile heart, we performed scRNA-seq on 386 FACS-purified cardiopharyngeal lineage cells dissociated from stage 38 juveniles, and identified clusters corresponding to pharyngeal muscle and cardiac lineages, including Mhc2/Myh6+ cardiomyocytes and an Mhc2/Myh6- population that expressed the second heart lineage markers Dach and Matn (Fig. 3h, Extended Data Fig. 7f-g). Triple labeling with Mesp 
and $T b x 1 / 10$ reporters indicated that these Dach+;Matn+ cells derived principally from the second heart precursors and formed the outer layer of the juvenile heart (Fig. 3i, Extended Data Fig. 7d), suggesting that cellular diversity in the beating heart emerges from the initial segregation of the first and second heart lineages. Consistent with this hypothesis, CRISPR/Cas9-mediated loss of Dach and Tbx1/10 early functions increased the proportions of Mhc2/Myh6+ cells in the SHP progeny (Fig. 3g, Extended Data Fig. 7b), demonstrating that early inhibition of the Mmp21+ first-lineage-specific program by the Tbx1/10-Dach pathway limits the potential of second heart lineage derivatives to differentiate into Mhc2/Myh6+ cardiomyocytes during organogenesis.

\section{Conserved cardiopharyngeal transcriptional signatures in chordates}

Finally, we asked whether molecular features of cardiopharyngeal development are shared between Ciona and vertebrates. Recent scRNA-seq analysis of early mesodermal lineages in mice identified a population of pharyngeal mesoderm marked by high levels of both Tbx1 and Dach1 expression ${ }^{54}$ (Fig. 4a). Multicolor immunohistochemical staining revealed that Dach1 expression starts broadly in the pharyngeal mesoderm, and becomes restricted to second heart field cells in the dorsal pericardial wall, and to a defined population of outflow tract cells, both of which also express Isl1 (Fig. 4b, Extended Data Fig. 8a-b). Dach1 expression was excluded from the Nkx2.5+ ventricle, and absent from the Isl1+ skeletal muscle progenitor cells in the core mesoderm of the first and second pharyngeal arches (Fig. 4b, Extended Data Fig. 8a-b), in a manner reminiscent of Dach exclusion from the pharyngeal muscles in Ciona (Fig. 3d-e, Extended Data Fig. 1f).

We extended the Ciona-to-mouse comparison of cardiopharyngeal transcriptomes using published scRNA-seq datasets ${ }^{13,54,55}$. Using canonical correlation analysis ${ }^{56}$, we identified genes that separated cardiac and pharyngeal mesoderm cells in both Ciona and E8.25 mouse embryos (Fig. 4c, Extended Data Table 5). We then used only the 30 best correlated genes to re-cluster scRNA-seq data independently from each species, and found that these markers sufficed to distinguish cardiac and pharyngeal muscle cells in either species, revealing a shared transcriptional program (Fig. 4c). We repeated this analysis using mouse datasets from earlier embryonic stages ${ }^{13,54}$, and consistently identified genes that separated cardiac and pharyngeal cells in both species, and were enriched in transcription factor- and DNA binding protein-coding genes (Extended Data Figs. 9a-b, 10a-b, 11). For instance, Gata4 and Ebf1 homologs were identified in all three comparisons as discriminating markers that separated cardiac and pharyngeal cells (Extended Data Fig. 10c, Extended Data Table 5). Overall, this analysis suggests that an evolutionary conserved transcriptional program, comprising homologs of Ebf1, Gata4 and other regulatory genes, govern the heart vs. pharyngeal muscle fate choice in cardiopharyngeal mesoderm.

\section{DISCUSSION}

Here, we present an extensive analysis of the transcriptome dynamics underlying early cardiopharyngeal development in a tractable chordate model. Using established clonal relationships to inform the reconstruction of developmental trajectories, we characterized essential features of the transcriptome dynamics underlying cardiopharyngeal multipotency and 
early fate specification. Multipotent cardiopharyngeal progenitors exhibit extensive multilineage transcriptional priming, although de novo gene activation significantly contributes to cell-type-specific transcriptomes, highlighting the importance of both transcriptional and post-transcriptional regulation in early heart vs. pharyngeal muscle fate choices.

Both first- and second-heart-lineage cells acquire a cardiac identity as they down-regulate multipotent progenitors markers and primed pharyngeal muscle genes, and deploy the full pan-cardiac program, entering this 'transition state ${ }^{36}$ upon termination of FGF-MAPK signaling. We propose that the dual functions of FGF-MAPK signaling, as observed during early cardiac specification in Ciona, are conserved in vertebrates considering that FGF-MAPK inputs are necessary to induce multipotent progenitors ${ }^{57-59}$, whereas signal termination is required for subsequent commitment to a heart fate and cardiomyocyte differentiation ${ }^{60-65}$.

Following commitment to a cardiac identity, first heart progenitors transition to an $\mathrm{Mmp21+}$ state that precedes differentiation into Mhc2/Myh6+ cardiomyocytes in the beating heart. By contrast, second heart progenitors activate Dach in response to Tbx1/10 inputs inherited from their distinct multipotent mother cells. This Tbx1/10-Dach pathway inhibits Mmp21 expression and the Mhc2/Myh6+ cardiomyocyte potential to foster heart cell diversity. As is the case in vertebrates $^{41,51,52,66-69}$, Tbx1/10 plays a dual roles in branchiomeric/pharyngeal myogenesis ${ }^{24,31,35}$ and second heart lineage development, thus acting as a bona fide regulator of cardiopharyngeal multipotency. Moreover, the first and second heart precursors share a common cardiac identity but differ because they emerge from successive multipotent progenitors before and after the onset of Tbx1/1O expression. Cellular diversity in the Ciona heart thus emerges by temporal patterning, as is the case for neuronal and muscle fates in Drosophila ${ }^{70,71}$. The proposal that first and second heart lineages form a coherent cardiac developmental unit, whereas distinct lineages contribute to cellular diversity within the heart, reconciles different views about the significance of the second heart field.

Finally, by leveraging recent computational methods for cross-species comparisons of single cell RNA-seq datasets, we identified shared markers of cardiopharyngeal regulatory states, while highlighting differences in expression dynamics, as seen for Dach homologs, thus illustrating the plasticity of gene regulatory networks controlling conserved developmental programs.

\section{ACKNOWLEDGEMENTS}

We are grateful to Florian Razy-Krajka for discussions and sharing reagents prior to publication. We thank Ashley Powers for help processing the single cell samples in the early phase of this study, Christopher Hafemeister and Andrew Butler for discussion and help on computational analyses. This project was funded by NIH/NHLBI Ro1 award HL108643 to L.C., trans-Atlantic network of excellence award 15CVDo1 from the Leducq Foundation to R.K. and L.C., and an NIH New Innovator Award (DP2-HG-009623) to R.S.

\section{AUTHORS' CONTRIBUTIONS}

W.W. performed the Ciona experiments. E.J. performed the mouse experiments. X.N., T.S., and R.S. performed computational analyses. W.M.M., W.W., and R.S. performed the single cell RNA-seq experiments. W.W., X.N., R.K., R.S., and L.C. designed the experiments and analyses. W.W., X.N., R.S. and L.C. wrote the paper. 


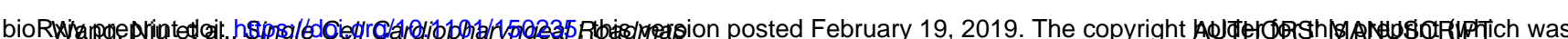
not certified by peer review) is the author/funder, who has granted bioRxiv a license to display the preprint in perpetuity. It is made available under aCC-BY-NC-ND 4.0 International license.

COMPETING FINANCIAL INTERESTS

The authors declare no competing financial interests. 
a

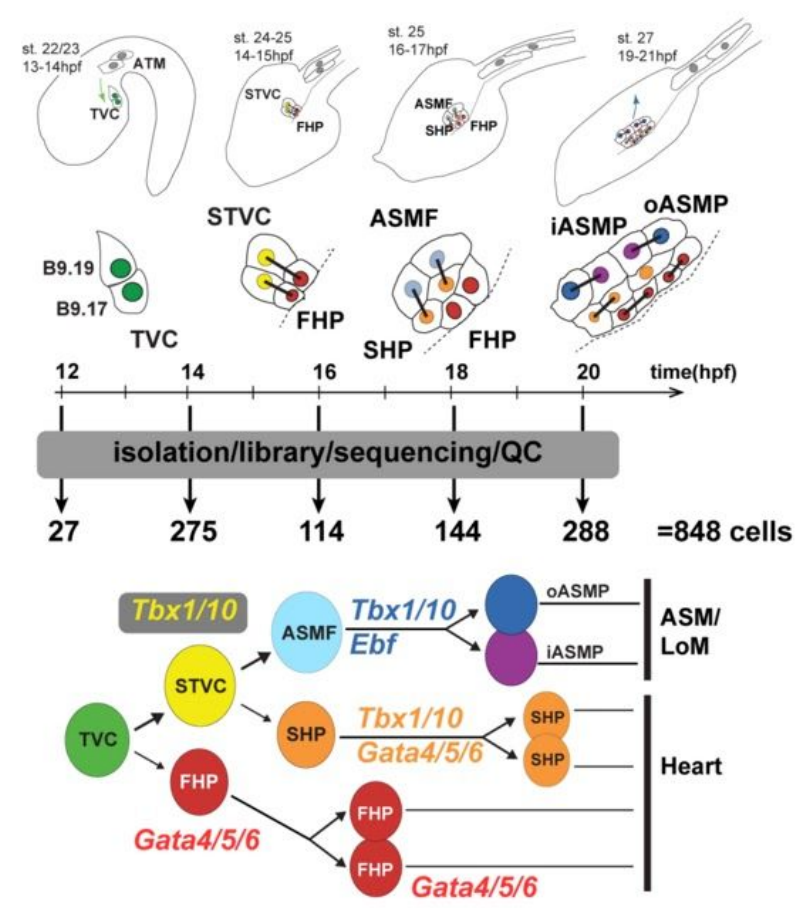

b

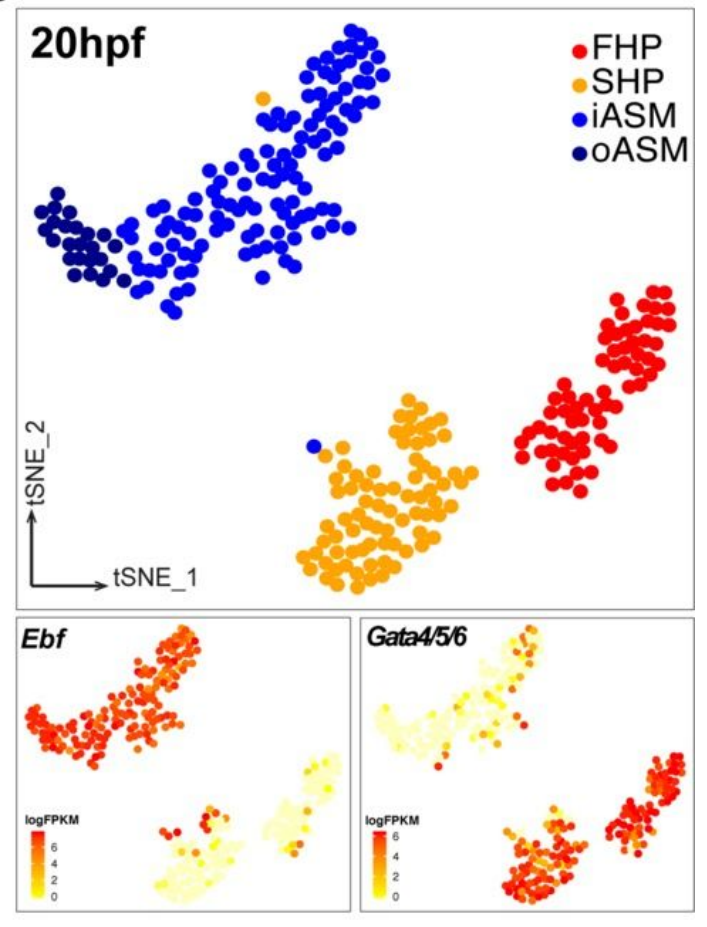

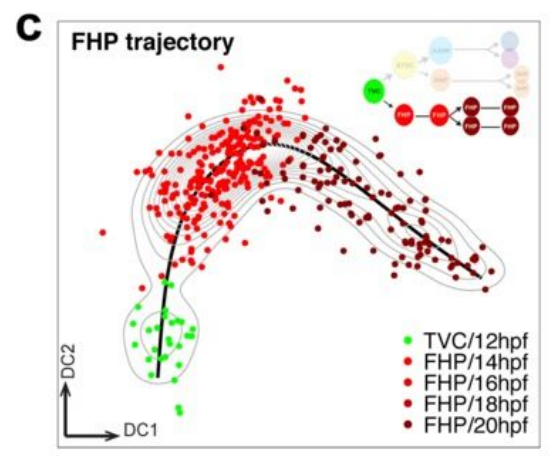

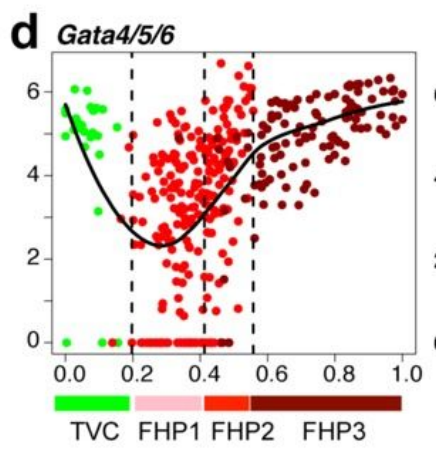

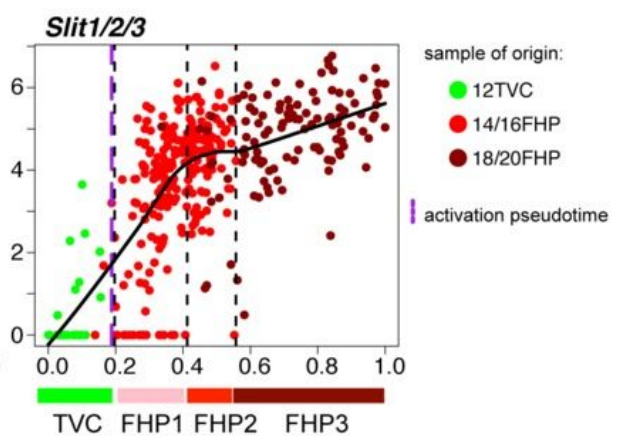

f

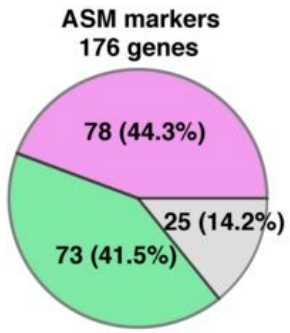

Whole transcriptome 5590 genes

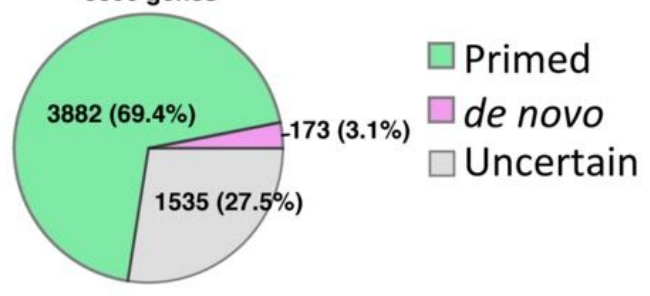


Figure 1|Cell clustering and pseudotemporal reconstruction of cardiopharyngeal developmental trajectories. (a) Early cardiopharyngeal development in Ciona, and sampling stages for scRNA-seq and established TVC lineage tree. Cardiopharyngeal lineage cells are shown for only one side and known cell-type-specific marker genes are indicated. st., stage according to ${ }^{72}$; hpf, hours post-fertilization; TVC, trunk ventral cell; STVC, second trunk ventral cell; FHP, first heart precursor; ASMF, atrial siphon muscle founder cells; SHP, second heart precursor; iASMP, inner atrial siphon muscle precursor; oASMP, outer atrial siphon muscle precursor, according to ${ }^{73}$; LoM, longitudinal muscles; QC, quality control. Dotted line: midline.

(b) t-distributed Stochastic Neighbor Embedding (t-SNE) plots of 20 hpf scRNA-seq data showing distinct clusters of progenitor subtypes: FHP (red), SHP (orange), iASM (blue) and oASM(dark blue). Indicated marker gene expression levels are color-coded and shown on corresponding clusters.

(c) 2D diffusion map of single cell transcriptomes for the FHP developmental trajectory. Black lines: principal curve; light gray contours: single cell density distribution. Color codes correspond to assigned cell identities following clustering at each time point. hpf, hours post-fertilization. Cartoon shows the cells used to reconstruct the unidirectional FHP trajectory.

(d) Pseudotemporal expression profiles of indicated genes along the FHP trajectory. X-axis: normalized pseudotime as defined in (e), Y-axes: expression in $\log _{2}$ FPKM. Black lines indicate the smoothed expression. Black dashed lines indicate the transitions between predicted regulatory states, indicated and color-coded below, and purple dashed lines indicate calculated activation pseudotime. Dot colors refer to the sample of origin as indicated in (c).

(e) Cross-correlation heatmaps to infer regulatory states along the FHP trajectory. Dendrogram (left) obtained from constrained hierarchical clustering. Top bars indicate the sample of origin with color codes as in (c). PCC, Pearson Correlation Coefficient.

(f) Proportions of primed vs. de novo genes among defined categories of marker genes. 

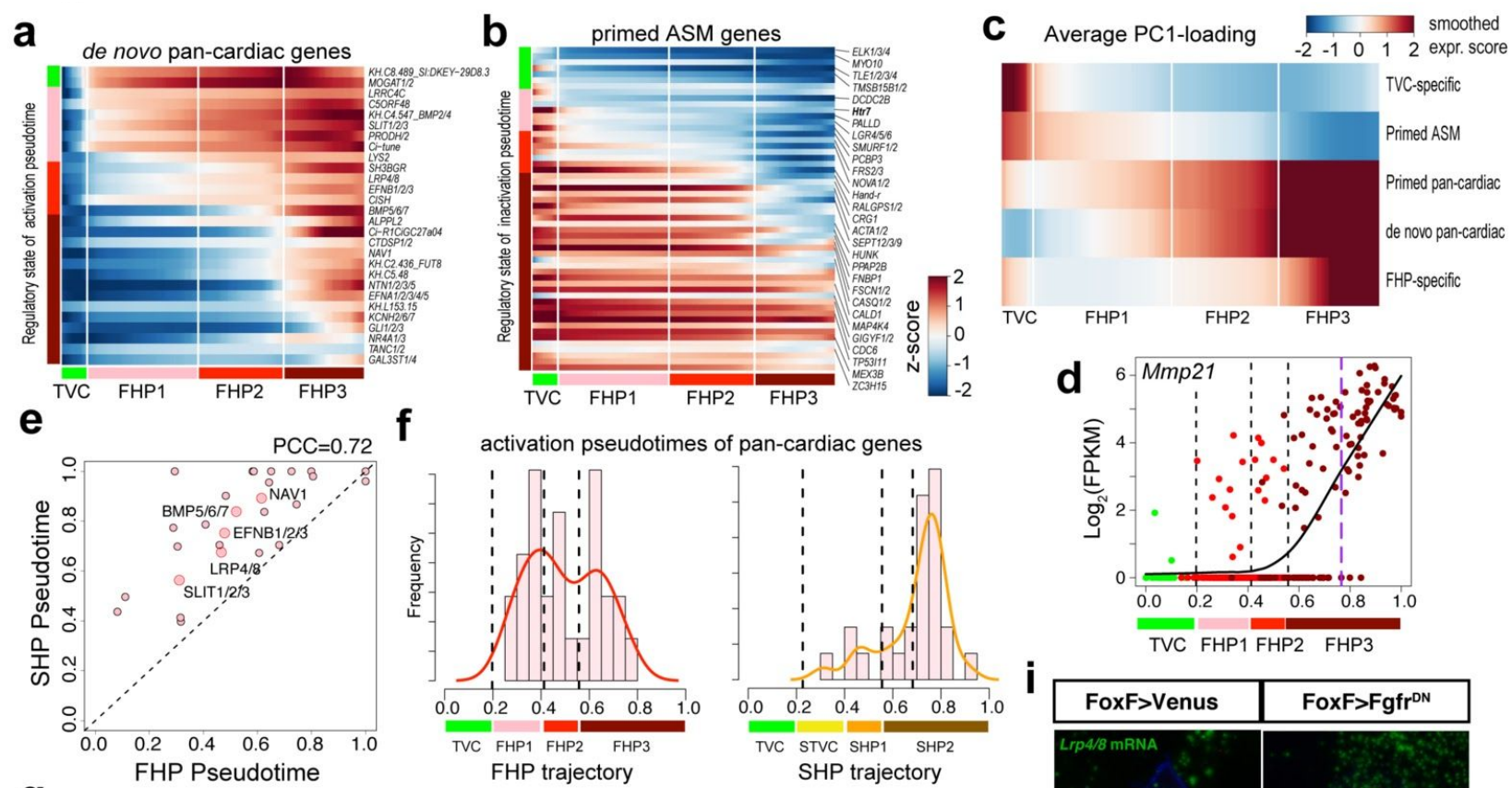

g

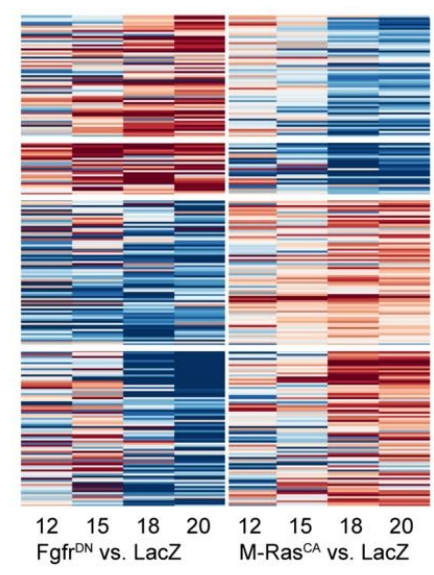

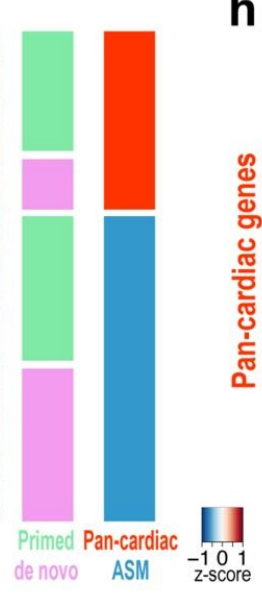
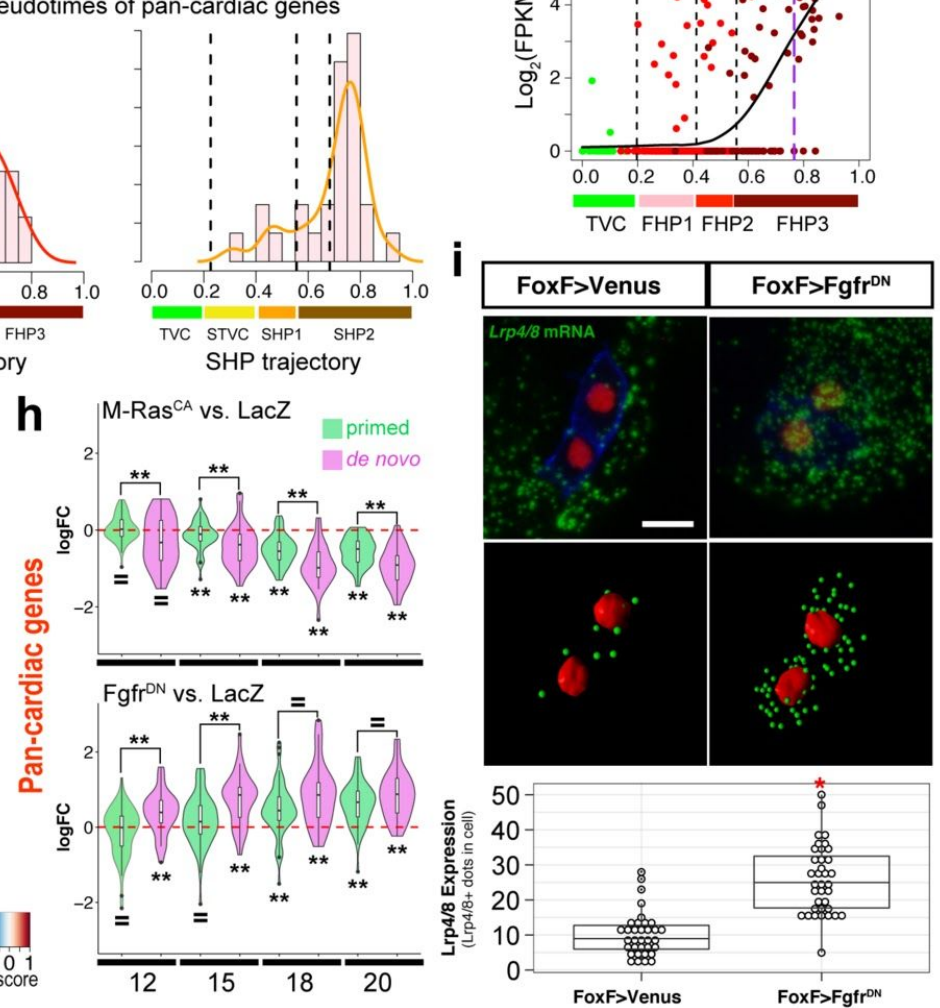

Figure 2| Characterization of the pan-cardiac gene expression program.

(a) Heatmap of smoothed gene expression showing the successive activations of de novo-expressed pan-cardiac genes.

(b) Heatmap of smoothed gene expression showing progressive depletion of primed ASM genes. For (a) and (b), white vertical lines indicate predicted regulatory states and color bar indicates relative expression level .

(c) Average PC1-loading scores per indicated gene category, mapped onto the FHP trajectory.

(d) Pseudotemporal expression profiles of Mmp21 along the FHP trajectory. X-axis: normalized pseudotime. Black dashed lines: transitions between regulatory states; purple dashed line: calculated activation pseudotime. Dots colors refer to the sample of origin as in Fig. 1c.

(e) Activation pseudotimes for de novo-expressed pan-cardiac genes along the FHP and SHP pseudotime axes. PCC: Pearson's Correlation Coefficient.

(f) Proportions of de novo pan-cardiac genes with calculated activation pseudotime in binned pseudotime windows along FHP and SHP trajectory respectively.

(g) Log fold changes of primed/de novo ASM and pan-cardiac genes in Fgfr ${ }^{\mathrm{DN}}$ vs. LacZ and M-Ras ${ }^{\mathrm{CA}}$ vs. LacZ pairwise comparisons from FACS-purified cardiopharyngeal lineage cells obtained from 12, 15, 18 and 20 hpf larvae.hpf: hours post-fertilization. 
(h) Distributions of $\log _{2}$ fold changes in indicated conditions and time points relative to LacZ controls, and parsed by primed or de novo-expressed pan-cardiac genes. Summary statistics: results of t-test for significant difference from o are indicated below violin plots, results for KS tests for significant differences between "Primed" and "De novo" gene sets in each condition are indicated above violin plots. "=", no difference, “**”, P-value <0.01. Details in Extended Data Table 4.

(i) FGF/MAPK inhibition induces precocious expression of $\operatorname{Lrp} 4 / 8$ in multipotent cardiopharyngeal progenitors (TVCs). Lrp $4 / 8$ mRNAs (green) visualized by FISH. Anti beta-galactosidase antibody (red) marks TVC nuclei expressing Mesp $>n l s:$ LacZ. Mesp $>\mathrm{hCD} 4:$ :mCherry, revealed by anti mCherry antibody (blue), marks cell membranes. Anterior to the left. Scale bar, $10 \mu \mathrm{m}$. Imaris-processed images show $\mathrm{Lrp} 4 / 8$ expression in the TVCs. Box plots represent the distributions of numbers of $L r p 4 / 8+$ dots per cell in indicated conditions. Bars in the box indicate the median value. ${ }^{*} \mathrm{p}<0.05$ (Student's t-test). 
a

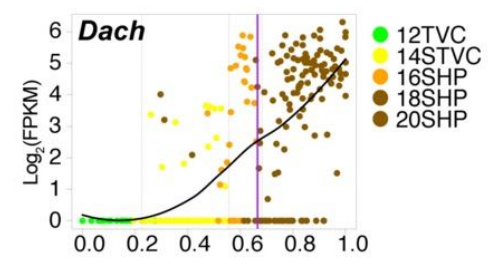

C Mmp21 expression

$\square$ Restricted in FHPs (Wt) $=$ Ectopic in $1-3 \mathrm{SHPs}=$ Ectopic in all SHPS

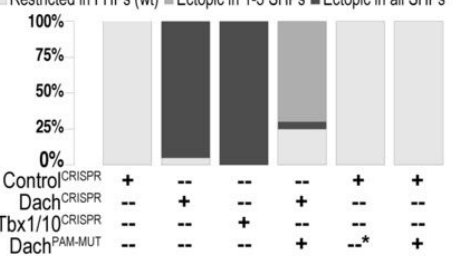

b
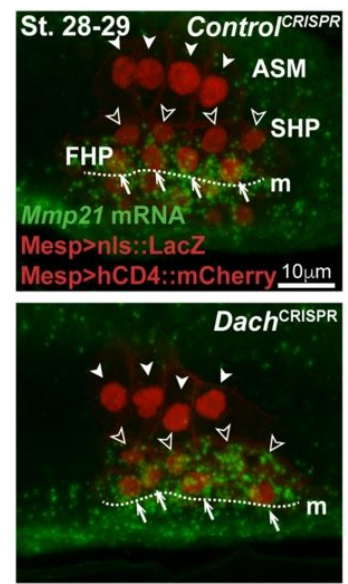

d
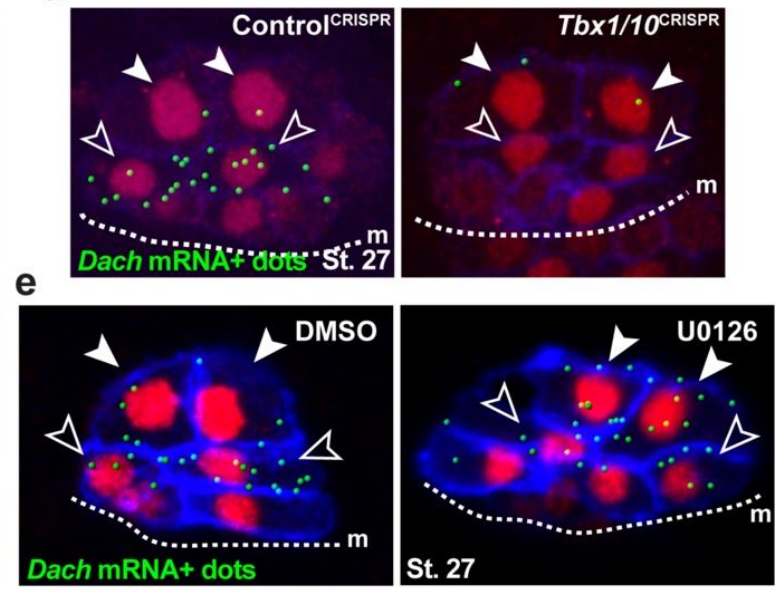

f
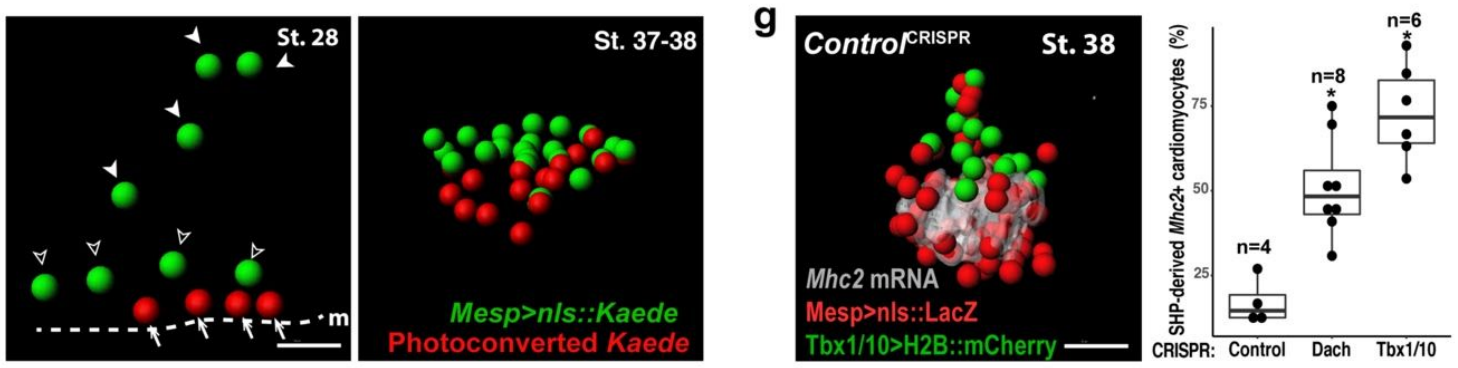

h
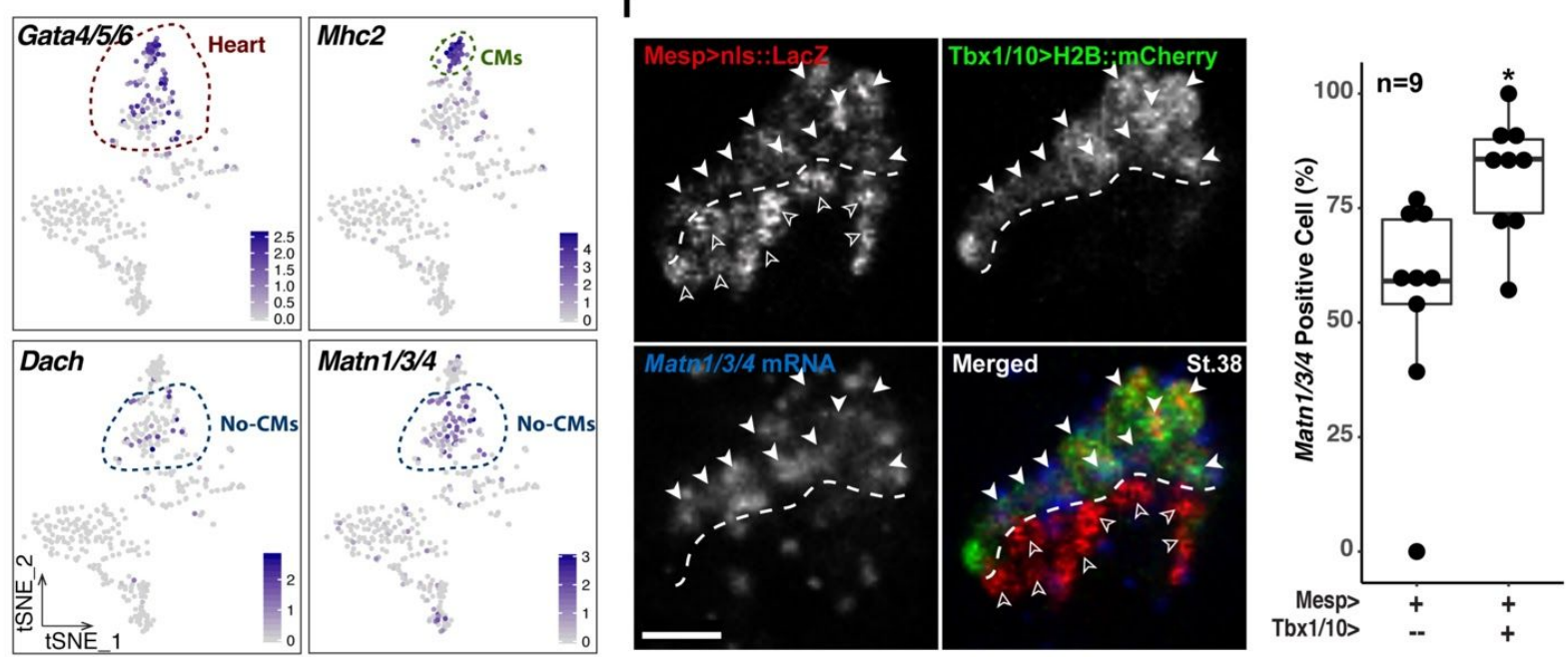

Figure 3| Transcriptional regulation of SHP fate specification.

(a) Dach pseudotemporal expression pattern on the SHP trajectory. Black dashed lines: transitions between SHP regulatory states defined in Ext. Data Fig. 3h,j; purple dashed line: predicted induction time .

(b) CRISPR/Cas9-mediated mutagenesis of Dach causes ectopic expression of the FHP-specific gene Mmp21 in the SHPs. Mmp21 mRNAs (green) detected by FISH. Mesp $>n l s:: L a c Z$, revealed by anti beta-galactosidase antibody (red), marks the nuclei of cardiopharyngeal lineage cells. Mesp $>h C D 4:: m C h e r r y$, revealed by anti-mCherry antibody (red), marks cell membranes. Open arrowheads, ASMPs; solid arrowheads, SHPs; arrows, FHPs. Dotted line: ventral midline. Anterior to the left. Scale bar, $10 \mu \mathrm{m}$.

(c) Proportions of larvae showing indicated phenotypes in each experimental condition. Wt, wild-type, indicates Mmp21 expression restricted to the FHPs. Dach ${ }^{\text {PAMmut }}$, Dach cDNA with wobble base mutation in the PAMs, driven by 
TVC-specific Foxf enhancer, renders the rescue construct (Foxf $>$ Dach ${ }^{\text {PAMmut }}$ ) resistant to CRISPR. *, The Dach ${ }^{\text {PAMmut }}$ control was electroporated with a neutral $F o x f>$ Venus construct.

(d) Tbx1/1O is required for Dach expression in SHP. B7.5-lineage-specific Dach expression visualized by FISH and shown as segmented Dach+ green dots (see Ext. Data Fig. 6 for raw images). Mesp $>n l s:$ LacZ, revealed with an anti beta-galactosidase antibody (red), labels nuclei of B7.5 lineage cells. Mesp>hCD4::mCherry, revealed by anti mCherry antibody (blue), marks cell membrane. Experiment performed in biological replicates. For each replicate, confocal stacks were acquired for 10 larvae in each condition. None of the $20 \mathrm{Tbx} 1 / 10^{\mathrm{CRISPR}}$ larvae showed Dach expression in SHPs. Solid arrowheads, ASMFs, open arrowheads, SHPs. Anterior to the left. Scale bar, $10 \mu \mathrm{m}$.

(e) FGF-MAPK signaling negatively regulates Dach expression in Tbx1/1O+ ASMFs. Representative Imaris processed confocal stacks showing Dach expression in 18.5hpf (St. 27) larvae, following 3.5 hours treatments with DMSO (control) or Uo126 (MEK inhibitor). Blocking MEK activity causes ectopic Dach expression in the ASMFs (solid arrowheads), in addition to its endogenous expression in the SHPs (open arrowheads) in 10/10 embryos analyzed.

(f) Photoconversion and lineage tracing of TVC progeny. Segmented nuclei of live B7.5 lineage cells labelled with Mesp>nls::Kaede::nls (green). Nuclear Kaede photoconverted to red specifically in the FHPs in a 16hpf (St.25) larva (see Ext. Data Fig. 7 for raw images). The same animal is shown at 65hpf (St.37-38). Open arrowheads, ASMPs; solid arrowheads, SHPs; arrows FHPs; Dotted line: ventral midline. Anterior to the left. Scale bar, $10 \mu \mathrm{m}$.

(g) Dach and Tbxi/1O activities inhibit the production of Mhc2+ cardiomyocytes from the second heart lineage. Rendered segmented signals are shown (see Methods). Grey: Mhc2 mRNA FISH. SHP-derived cells are labelled with the Tbx1/10 reporter, 3XT12>H2B::mCherry revealed by an anti-mCherry antibody (green), B7.5 lineage cells are labelled with Mesp $>$ nls::LacZ (red). Proportions of Mhc2+ cells among the 3xT12>H2B::mCherry+ SHP-derived cells in juvenile hearts in indicated experimental conditions. Scale bar, $10 \mu \mathrm{m}$.

(h) t-SNE plots of Ciona scRNA-seq data acquired in FACS-purified cardiopharyngeal lineage cells from 72hpf juveniles (St. 38), with expression pattern of Gata4/5/6, Mhc2, Dach and Matn1/3/4 (see Ext. Data fig. 7f,g for clusters and top markers). The red dash line indicates two Gata4/5/6+ heart cell clusters. The green dash line indicates the cluster of Mhc2+ cardiomyocytes (CM). The blue dash line highlights the cluster of Dach+ and Matn1/3/4+ non-cardiomyocyte (No-CM) cells.

(i) Matn1/3/4 is enriched in the SHP-derived Tbx1/1O(3xT12)>H2B::mCherry+ cells (green). Matn1/3/4 mRNAs (Blue) are visualized by FISH. Mesp $>n l s:: L a c Z$, revealed by anti beta-galactosidase antibody (red), marks the nuclei of cardiopharyngeal lineage cells. Solid arrowheads (outer-layer): mCherry+/beta-galactosidase+ SHP-derived cells positive for Matn1/3/4 mRNA, open arrowheads (inner layer): mCherry-/beta-galactosidase+ cells negative for Matn1/3/4 expression. Scale bar, $5 \mu \mathrm{m}$. The images are X - Y cross sections of the juvenile heart. Corresponding boxplots with the proportions of Matn1/3/4+ cells among the Mesp>nls::LacZ+;3xT12>H2B::mCherry+ SHP-derived cells in juvenile hearts. There are significantly more Matn1/3/4+ cells among SHP-derived cells, compared to the Mesp $>$ nls::LacZ+;Tbx1/10(3xT12) $>\mathrm{H} 2 \mathrm{~B}:$ :mCherry- cells derived primarily from the first heart lineage. Median values are indicated in the boxplots. ${ }^{*} \mathrm{p}<0.05$ (Student's t-test) 


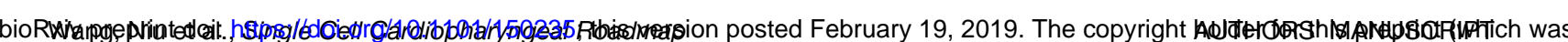
not certified by peer review) is the author/funder, who has granted bioRxiv a license to display the preprint in perpetuity. It is made available under aCC-BY-NC-ND 4.0 International license.

a
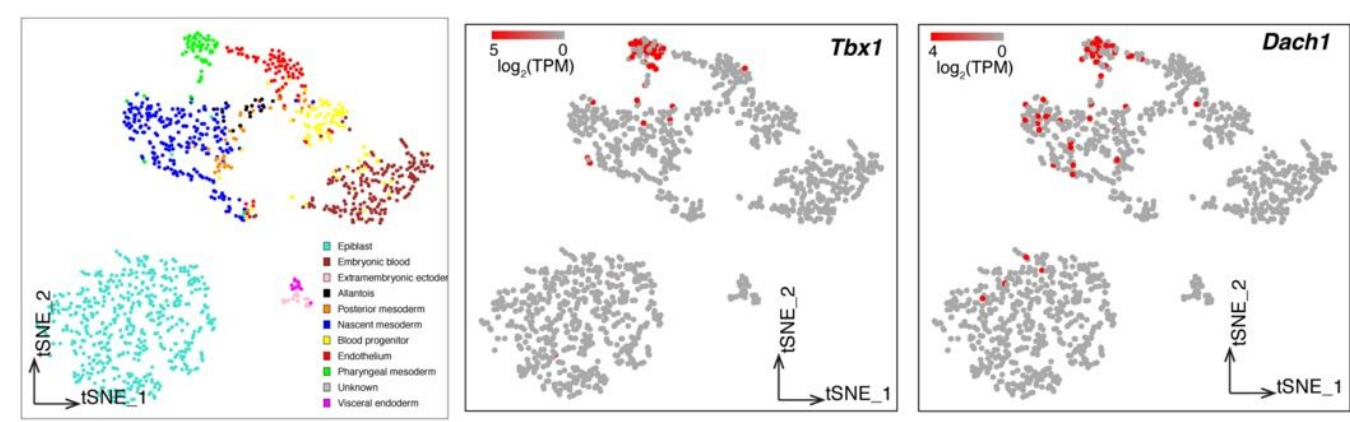

b
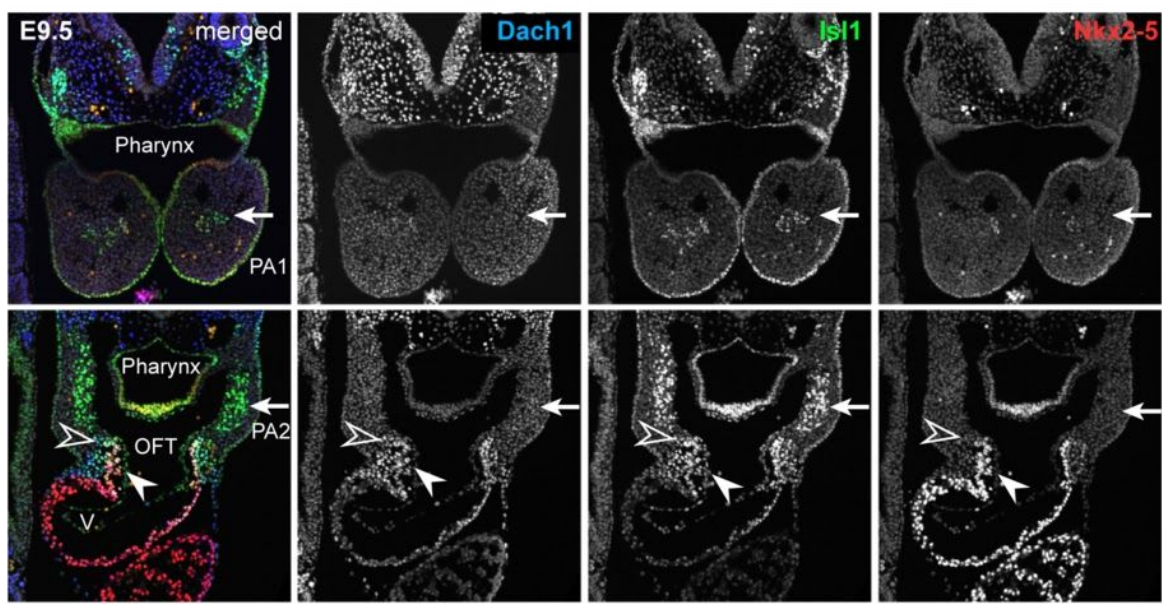

C
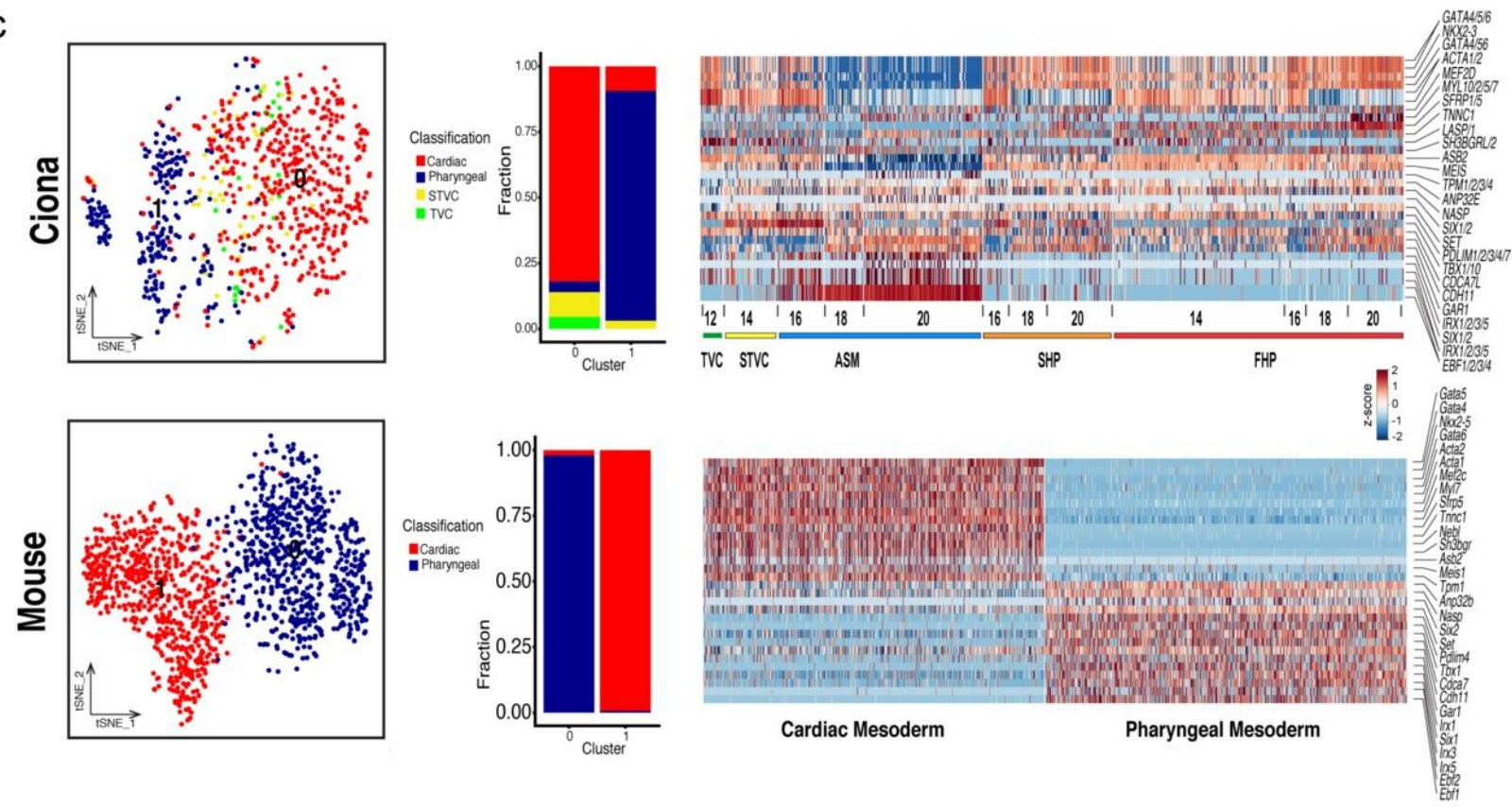

Figure 4| Conserved cardiopharyngeal programs in Ciona and in the Mouse.

(a) t-SNE plots of mouse scRNA-seq data after Scialdone et al. ${ }^{74}$, with expression pattern of $T b x 1$ and Dach1. Cluster identities are as determined in the original publication, with the pharyngeal mesoderm shown in green.

(b) Expression patterns of Dach1, Islet1 and Nkx2.5 proteins in E9.5 mouse embryos. Arrows: Islet1+ head muscle progenitor cells in the mesodermal core of the first (PA1, top) and second (PA2, bottom) pharyngeal arches, showing absence of Dach1 and Nkx2.5 expression. Open arrowhead: Dach1+, Islet1+, Nkx2.5- second heart field cells in the 
dorsal pericardial wall, solid arrowhead: Triple Nkx2.5+, Dach1+, Islet1+ second heart field-derived cells in the outflow tract (OFT). Note the Nkx2.5+, Dach1-, Islet1- cells in the ventricle (V).

(c) Aligned structure of Ciona and Mouse E8.25 cardiopharyngeal cells. tSNE plots showing the clustering of Ciona and Mouse E8.25 cardiopharyngeal cells, respectively, using conserved markers determined by canonical correlation (CC). Barplots indicate that original cell identities, defined in each species independently, as recovered in the clustering using conserved markers. Heatmaps show the single cell expression profiles for the top 30 conserved markers in each species, separately. 


\section{REFERENCES}

1. Pinto, A. R. et al. Revisiting Cardiac Cellular Composition. Circ. Res. 118, 400-409 (2016).

2. Meilhac, S. M., Lescroart, F., Blanpain, C. \& Buckingham, M. E. Cardiac cell lineages that form the heart. Cold Spring Harb. Perspect. Med. 5, a026344 (2015).

3. Saga, Y. et al. MesP1 is expressed in the heart precursor cells and required for the formation of a single heart tube. Development 126, 3437-3447 (1999).

4. Lescroart, F. et al. Early lineage restriction in temporally distinct populations of Mesp1 progenitors during mammalian heart development. Nat. Cell Biol. 16, 829-840 (2014).

5. Devine, W. P., Wythe, J. D., George, M., Koshiba-Takeuchi, K. \& Bruneau, B. G. Early patterning and specification of cardiac progenitors in gastrulating mesoderm. Elife $\mathbf{3}$, (2014).

6. Meilhac, S. M. et al. A retrospective clonal analysis of the myocardium reveals two phases of clonal growth in the developing mouse heart. Development 130, 3877-3889 (2003).

7. Meilhac, S. M., Esner, M., Kelly, R. G., Nicolas, J.-F. \& Buckingham, M. E. The clonal origin of myocardial cells in different regions of the embryonic mouse heart. Dev. Cell 6, 685-698 (2004).

8. Kelly, R. G., Brown, N. A. \& Buckingham, M. E. The arterial pole of the mouse heart forms from Fgf10-expressing cells in pharyngeal mesoderm. Dev. Cell 1, 435-440 (2001).

9. Waldo, K. L. et al. Conotruncal myocardium arises from a secondary heart field. Development 128, 3179-3188 (2001).

10. Mosimann, C. et al. Chamber identity programs drive early functional partitioning of the heart. Nat. Commun. 6, 8146 (2015).

11. Nevis, K. et al. Tbx1 is required for second heart field proliferation in zebrafish. Dev. Dyn. 242, 550-559 (2013).

12. Lescroart, F. et al. Early lineage restriction in temporally distinct populations of Mesp1 progenitors during mammalian heart development. Nat. Cell Biol. 16, 829-840 (2014).

13. Lescroart, F. et al. Defining the earliest step of cardiovascular lineage segregation by single-cell RNA-seq. Science 359, 1177-1181 (2018).

14. Diogo, R. et al. A new heart for a new head in vertebrate cardiopharyngeal evolution. Nature 520, 466-473 (2015).

15. Nathan, E. et al. The contribution of Islet1-expressing splanchnic mesoderm cells to distinct branchiomeric muscles reveals significant heterogeneity in head muscle development. Development 135, 647-657 (2008).

16. Harel, I. et al. Pharyngeal mesoderm regulatory network controls cardiac and head muscle morphogenesis. Proc. Natl. Acad. Sci. U. S. A. 109, 18839-18844 (2012).

17. Tirosh-Finkel, L., Elhanany, H., Rinon, A. \& Tzahor, E. Mesoderm progenitor cells of common origin contribute to the head musculature and the cardiac outflow tract. Development 133, 1943-1953 (2006).

18. Lescroart, F. et al. Clonal analysis reveals a common origin between nonsomite-derived neck muscles and heart myocardium. Proc. Natl. Acad. Sci. U. S. A. 112, 1446-1451 (2015).

19. Lescroart, F. et al. Clonal analysis reveals common lineage relationships between head muscles and second heart field derivatives in the mouse embryo. Development 137, 3269-3279 (2010).

20. Gopalakrishnan, S. et al. A Cranial Mesoderm Origin for Esophagus Striated Muscles. Dev. Cell 34, 694-704 (2015).

21. Mandal, A., Holowiecki, A., Song, Y. C. \& Waxman, J. S. Wnt signaling balances 
specification of the cardiac and pharyngeal muscle fields. Mech. Dev. 143, 32-41 (2017).

22. Chan, S. S.-K. et al. Development of Bipotent Cardiac/Skeletal Myogenic Progenitors from MESP1+ Mesoderm. Stem Cell Reports 6, 26-34 (2016).

23. Kaplan, N., Razy-Krajka, F. \& Christiaen, L. Regulation and evolution of cardiopharyngeal cell identity and behavior: insights from simple chordates. Curr. Opin. Genet. Dev. 32, 119-128 (2015).

24. Wang, W., Razy-Krajka, F., Siu, E., Ketcham, A. \& Christiaen, L. NK4 antagonizes Tbx1/10 to promote cardiac versus pharyngeal muscle fate in the ascidian second heart field. PLoS Biol. 11, e1001725 (2013).

25. Razy-Krajka, F. et al. Collier/OLF/EBF-dependent transcriptional dynamics control pharyngeal muscle specification from primed cardiopharyngeal progenitors. Dev. Cell 29, 263-276 (2014).

26. Picelli, S. et al. Smart-seq2 for sensitive full-length transcriptome profiling in single cells. Nat. Methods 10, 1096-1098 (2013).

27. Satija, R., Farrell, J. A., Gennert, D., Schier, A. F. \& Regev, A. Spatial reconstruction of single-cell gene expression data. Nat. Biotechnol. 33, 495-502 (2015).

28. Stolfi, A. et al. Early chordate origins of the vertebrate second heart field. Science 329, 565-568 (2010).

29. Trapnell, C. et al. The dynamics and regulators of cell fate decisions are revealed by pseudotemporal ordering of single cells. Nat. Biotechnol. 32, 381-386 (2014).

30. Bendall, S. C. et al. Single-Cell Trajectory Detection Uncovers Progression and Regulatory Coordination in Human B Cell Development. Cell 157, 714-725 (2014).

31. Razy-Krajka, F. et al. An FGF-driven feed-forward circuit patterns the cardiopharyngeal mesoderm in space and time. Elife 7, (2018).

32. Grimm, E. C. CONISS: a FORTRAN 77 program for stratigraphically constrained cluster analysis by the method of incremental sum of squares. Comput. Geosci. 13, 13-35 (1987).

33. Nimmo, R. A., May, G. E. \& Enver, T. Primed and ready: understanding lineage commitment through single cell analysis. Trends Cell Biol. 25, 459-467 (2015).

34. Graf, T. \& Enver, T. Forcing cells to change lineages. Nature 462, 587-594 (2009).

35. Tolkin, T. \& Christiaen, L. Rewiring of an ancestral Tbx1/10-Ebf-Mrf network for pharyngeal muscle specification in distinct embryonic lineages. Development 143, 3852-3862 (2016).

36. Moris, N., Pina, C. \& Arias, A. M. Transition states and cell fate decisions in epigenetic landscapes. Nat. Rev. Genet. 17, 693-703 (2016).

37. Prall, O. W. J. et al. An Nkx2-5/Bmp2/Smad1 negative feedback loop controls heart progenitor specification and proliferation. Cell 128, 947-959 (2007).

38. Zhang, L. et al. Mesodermal Nkx2.5 is necessary and sufficient for early second heart field development. Dev. Biol. 390, 68-79 (2014).

39. Grifone, R. \& Kelly, R. G. Heartening news for head muscle development. Trends Genet. 23, 365-369 (2007).

40. Chen, L., Fulcoli, F. G., Tang, S. \& Baldini, A. Tbx1 regulates proliferation and differentiation of multipotent heart progenitors. Circ. Res. 105, 842-851 (2009).

41. Liao, J. et al. Identification of downstream genetic pathways of Tbx1 in the second heart field. Dev. Biol. 316, 524-537 (2008).

42. Chen, R., Amoui, M., Zhang, Z. \& Mardon, G. Dachshund and Eyes Absent Proteins Form a Complex and Function Synergistically to Induce Ectopic Eye Development in Drosophila. Cell 91, 893-903 (1997).

43. Davis, R. J., Shen, W., Heanue, T. A. \& Mardon, G. Mouse Dach, a homologue of 
Drosophila dachshund, is expressed in the developing retina, brain and limbs. Dev. Genes Evol. 209, 526-536 (1999).

44. Heanue, T. A. et al. Dach1, a vertebrate homologue of Drosophila dachshund, is expressed in the developing eye and ear of both chick and mouse and is regulated independently of Pax and Eya genes. Mech. Dev. 111, 75-87 (2002).

45. Davis, R. J., Shen, W., Sandler, Y. I., Heanue, T. A. \& Mardon, G. Characterization of mouse Dach2, a homologue of Drosophila dachshund. Mech. Dev. 102, 169-179 (2001).

46. Kumar, J. P. The molecular circuitry governing retinal determination. Biochim. Biophys. Acta 1789, 306-314 (2009).

47. Guo, C. et al. A Tbx1-Six1/Eya1-Fgf8 genetic pathway controls mammalian cardiovascular and craniofacial morphogenesis. J. Clin. Invest. 121, 1585-1595 (2011).

48. Zhou, Z. et al. Temporally Distinct Six2-Positive Second Heart Field Progenitors Regulate Mammalian Heart Development and Disease. Cell Rep. 18, 1019-1032 (2017).

49. Stolfi, A., Gandhi, S., Salek, F. \& Christiaen, L. Tissue-specific genome editing in Ciona embryos by CRISPR/Cas9. Development 141, 4115-4120 (2014).

50. Gandhi, S., Haeussler, M., Razy-Krajka, F., Christiaen, L. \& Stolfi, A. Evaluation and rational design of guide RNAs for efficient CRISPR/Cas9-mediated mutagenesis in Ciona. Dev. Biol. (2017). doi:10.1016/j.ydbio.2017.03.003

51. Kelly, R. G., Jerome-Majewska, L. A. \& Papaioannou, V. E. The del22q11.2 candidate gene Tbx1 regulates branchiomeric myogenesis. Hum. Mol. Genet. 13, 2829-2840 (2004).

52. Kong, P. et al. Tbx1 is required autonomously for cell survival and fate in the pharyngeal core mesoderm to form the muscles of mastication. Hum. Mol. Genet. 23, 4215-4231 (2014).

53. Anderson, H. E. \& Christiaen, L. Ciona as a Simple Chordate Model for Heart Development and Regeneration. J Cardiovasc Dev Dis 3, (2016).

54. Scialdone, A. et al. Resolving early mesoderm diversification through single-cell expression profiling. Nature 535, 289-293 (2016).

55. Ibarra-Soria, X. et al. Defining murine organogenesis at single-cell resolution reveals a role for the leukotriene pathway in regulating blood progenitor formation. Nat. Cell Biol. 2o, 127-134 (2018).

56. Butler, A., Hoffman, P., Smibert, P., Papalexi, E. \& Satija, R. Integrating single-cell transcriptomic data across different conditions, technologies, and species. Nat. Biotechnol. 36, 411-420 (2018).

57. Abu-Issa, R., Smyth, G., Smoak, I., Yamamura, K.-I. \& Meyers, E. N. Fgf8 is required for pharyngeal arch and cardiovascular development in the mouse. Development 129, 4613-4625 (2002).

58. Barron, M., Gao, M. \& Lough, J. Requirement for BMP and FGF signaling during cardiogenic induction in non-precardiac mesoderm is specific, transient, and cooperative. Dev. Dyn. 218, 383-393 (2000).

59. Reifers, F., Walsh, E. C., Léger, S., Stainier, D. Y. \& Brand, M. Induction and differentiation of the zebrafish heart requires fibroblast growth factor 8 (fgf8/acerebellar). Development 127, 225-235 (2000).

60. Tirosh-Finkel, L. et al. BMP-mediated inhibition of FGF signaling promotes cardiomyocyte differentiation of anterior heart field progenitors. Development 137, 2989-3000 (2010).

61. Hutson, M. R. et al. Arterial pole progenitors interpret opposing FGF/BMP signals to proliferate or differentiate. Development 137, 3001-3011 (2010).

62. Marques, S. R., Lee, Y., Poss, K. D. \& Yelon, D. Reiterative roles for FGF signaling in the establishment of size and proportion of the zebrafish heart. Dev. Biol. 321, 397-406 
(2008).

63. van Wijk, B. et al. Epicardium and myocardium separate from a common precursor pool by crosstalk between bone morphogenetic protein- and fibroblast growth factor-signaling pathways. Circ. Res. 105, 431-441 (2009).

64. Watanabe, Y. et al. Fibroblast growth factor 10 gene regulation in the second heart field by Tbx1, Nkx2-5, and Islet1 reveals a genetic switch for down-regulation in the myocardium.

Proc. Natl. Acad. Sci. U. S. A. 109, 18273-18280 (2012).

65. Zhang, J. et al. Frs2alpha-deficiency in cardiac progenitors disrupts a subset of FGF signals required for outflow tract morphogenesis. Development 135, 3611-3622 (2008).

66. Chen, L., Fulcoli, F. G., Tang, S. \& Baldini, A. Tbx1 regulates proliferation and differentiation of multipotent heart progenitors. Circ. Res. 105, 842-851 (2009).

67. Kelly, R. G. \& Papaioannou, V. E. Visualization of outflow tract development in the absence of Tbx1 using an FgF10 enhancer trap transgene. Dev. Dyn. 236, 821-828 (2007).

68. Vitelli, F., Morishima, M., Taddei, I., Lindsay, E. A. \& Baldini, A. Tbx1 mutation causes multiple cardiovascular defects and disrupts neural crest and cranial nerve migratory pathways. Hum. Mol. Genet. 11, 915-922 (2002).

69. Zhang, Z., Huynh, T. \& Baldini, A. Mesodermal expression of Tbx1 is necessary and sufficient for pharyngeal arch and cardiac outflow tract development. Development 133, 3587-3595 (2006).

70. Li, X. et al. Temporal patterning of Drosophila medulla neuroblasts controls neural fates. Nature 498, 456-462 (2013).

71. Dubois, L., Frendo, J.-L., Chanut-Delalande, H., Crozatier, M. \& Vincent, A. Genetic dissection of the Transcription Factor code controlling serial specification of muscle identities in Drosophila. Elife 5, (2016).

72. Hotta, K. et al. A web-based interactive developmental table for the ascidian Ciona intestinalis, including 3D real-image embryo reconstructions: I. From fertilized egg to hatching larva. Dev. Dyn. 236, 1790-1805 (2007).

73. Razy-Krajka, F. et al. Collier/OLF/EBF-dependent transcriptional dynamics control pharyngeal muscle specification from primed cardiopharyngeal progenitors. Dev. Cell 29, 263-276 (2014).

74. Scialdone, A. et al. Resolving early mesoderm diversification through single-cell expression profiling. Nature 535, 289-293 (2016). 